\title{
D-dimer/Fibrinogen ratio and recurrent exacerbations might have a potential impact to predict 90-day mortality in patients with COPD exacerbation
}

\author{
Cihan Aydin ${ }^{1}$, Birsen Pınar Yıldız ${ }^{*}$, Didem Görgün Hattatoğlu ${ }^{3}$ \\ 1. University of Health Sciences, Yedikule Chest Disease and Thoracic Surgery Training and Research Hospital, İstanbul, Turkey \\ 2. University of Health Sciences, Yedikule Chest Disease and Thoracic Surgery Training and Research Hospital, İstanbul, Turkey \\ 3. University of Health Sciences, Yedikule Chest Disease and Thoracic Surgery Training and Research Hospital, İstanbul, Turkey
}

*Correspondance: Birsen Pınar Yıldız; Email: pinary70@yahoo.com

\section{Background}

\section{Abstract}

According to the World Health Organisation reports (WHO), COPD is the third leading cause of overall in the World by 2020.

Aim

We aimed to determine the prognostic predictors of 90-day mortality after an initial exacerbation in patients with acute exacerbation of COPD (AECOPD).

Results

Increased Charlson Comorbidity Score(CCS) (HR:1.47; p<0.05), readmission after initial exacerbation (HR:1.47; p<0.05) were predictive risk factors for 30-day mortality in multivariable regression model. The 90 -day mortality rate was \%11.8. Hypertension, increased median age, nutrition risk score (NRS), CCS, CAT score, and mMRC 4th level were possible risk factors for 90-day mortality. There was a significant difference in the mortality of patients with D-dimer/Fibrinogen ratios $>0.11$ and $\leq 0.11$ (HR:2.47; p $<0.05$ ) Recurrent exacerbations after discharge were predictive risk factors for 90-day mortality in the multivariable regression model (HR:2.25; $\mathrm{p}<0.001)$ with the increased mortality risk 4.73 times (HR:4.73; $\mathrm{p}=0.002)$. Furthermore, a 1-unit increment of acute exacerbation increased the mortality risk 3.39 times (HR:3.39; $\mathrm{p}<0.001)$.

Conclusion

Our study showed that D-dimer/Fibrinogen ratio but not D-dimer and recurrent exacerbations after discharge might have a critical impact on 90-day mortality.

Keywords: COPD, D-dimer, exacerbation, mortality, prognosis

\section{Introduction}

According to the World Health Organisation reports (WHO), COPD is the third leading cause of overall in the World by $2020^{1}$. Acute exacerbation of COPD (AECOPD has a great impact on prognosis while associated with the severity and frequency ${ }^{2-4}$. Exacerbations have an increased risk of recurrence after an initial exacerbation and further deteriorate patients' health status ${ }^{5}$. The European Respiratory Society (ERS) reported high 90-day readmission rates with AECOPD across Europe about \%40. The time after the initial exacerbation of COPD may be a potential high-risk period for all-cause mortality ${ }^{7}$. Thus it needs to analyze possible prognostic factors associated with the 90day mortality which would be a vulnerable period after an initial exacerbation. It would be important to show poor prognostic features in patients with AECOPD to enhance the management of high-risk patients.

Hypercoagulability state may be induced by hypoxemia and carbon dioxide retention in $\mathrm{AECOPD}^{8}$. D-dimer is a fibrin degradation product (FDP) after a blood clot is degraded by fibrinolysis. It has been suggested as a prognostic marker in limited studies including patients with AECOPD which were not prospectively designed and/or without any precisely well-defined inclusion criteria-10. In a prospectively designed study, D-dimer $\geq 985 \mathrm{ng} / \mathrm{L}$ has been shown as an independent risk factor for both in-hospital and 1-year mortality. But they were not regarded as 90-day mortality. We aimed to determine the prognostic predictors of 90-day mortality which would be a vulnerable period after an initial exacerbation in patients with AECOPD.

We aim to determine the prognostic predictors of 90day mortality after an initial exacerbation in patients with AECOPD.

\section{Materials and Methods}

\section{Subjects}

We screened all patients with acute exacerbation of COPD, who were admitted and hospitalized in the pulmonology clinics of tertiary care hospital between 15 November 2019 and 15 November 2020. The diagnosis of COPD was decided by pulmonary physicians based on Global Initiative for Chronic Obstructive Lung Disease criterion (GOLD) 2020. The severity of disease was evaluated by the degree of decrease in FEV1: (a) FEV1 $\geq 80 \%$ predicted mild (GOLD-1); (b) 50\% $\leq$ FEV1 < 80\% predicted moderate (GOLD-2); 30\% $\leq$ FEV1 $<50 \%$ predicted severe (GOLD3 ), and FEV1 $<30 \%$ predicted extremely severe (GOLD$4)^{2}$. Patients rejected to sign the informed consent, having life-threatening severe illness including renal, liver and other organ failure did not include in the study. In addition, the 
diagnosis of CTEPH and acute pulmonary embolism in the admission has been accepted as an exclusion criterion. The ethics of Istanbul Training and Research Hospital approved the research protocol (approval number 2043, date 08.11.2019).

\section{Methods}

Patients' demographics including age, sex, the number of hospitalizations or admission to the emergency department for AECOPD in the previous year, smoking habit, and comorbidities were asked and recorded by the investigator. All medical records and laboratory results have been extracted from the hospital-based data system. The results of mMRC, pulmonary function test (PFT) results and 90-day mortality. Kaplan-Meier survival curves and log-rank tests were used to compare the time to death between those with elevated D-dimer levels and those without. The results are presented as hazard ratios (HRs) with a 95\% confidence interval (CI). We analyzed the data using SPSS 13.0 for Windows 20(SPSS Inc., Chicago, IL, USA). The p-value $<0.05$ was considered significant.

\section{Results}

Three hundred and sixty AECOPD patients were screened and 231 patients were included in our study. The demographic data of the subjects were shown in Table 1.

\section{Table 1.Demographic Data}

CAT score were evaluated in the stable Demographic parameters (N:231)
period and, CCS, NRS, CURB-65, and

DECAF were evaluated and recorded Gender, n(\%)

in exacerbation periods.CURB-65 is Male

a score to evaluate the AECOPD for Female

physicians and it contains the letters of

the words in order "Confusion, BUN, Age (years)

Respiratory rate, Blood pressure" and $\mathrm{BMl}, \mathrm{kg} / \mathrm{m} 2$

Comorbidities, $n(\%)$

the number for age 65 .

Pneumoniae

DECAF is a score that is developec
by some physicians and used in some studies to determine the prognosis of

AECOPD. DECAF contains the letters of the words in order "Extended MRC Dyspnea Scale (eMRCD), Eosinopenia, Consolidation on chest X-ray, Accidemia and atrial Fibrillation".

Clinical data, vital signs, and arterial blood gases $(\mathrm{pH}$, arterial carbon dioxide Peptic ulcer

\begin{tabular}{|l|l|l|l|}
\hline Ischemic heart disease & $53(22.9)$ & Diabetes Mellitus & $51(22.1)$ \\
\hline Myocardial infarction & $6(2.6)$ & Congestive heart failure & $48(20.8)$ \\
\hline Atrial fibrilation & $18(7.8)$ & Chronic renal failure & $13(5.7)$ \\
\hline Bronchiectasis & $9(3.9)$ & $\begin{array}{l}\text { Obstructive sleep apnea } \\
\text { syndrome }\end{array}$ & $3(1.3)$ \\
\hline Peripheral vasculary disease & $2(0.9)$ & Interstitial lung diseases & $2(0.9)$ \\
\hline Peptic ulcer & $2(0.9)$ & Cerebrovascular disease & $1(0.4)$ \\
\hline
\end{tabular}
tension ( $\mathrm{PaCO} 2)$, arterial oxygen tension $(\mathrm{PaO} 2)$, and arterial oxygen

Table 2. The relationship between demographic data and 90-day mortality saturation) on admission were recorded. We collected the blood samples from each patient at the time of admission to the inpatient clinic of pulmonology for Gender, $n(\%)$ D-dimer and laboratory measurements (creatinine, blood urea nitrogen (BUN), platelets, hemoglobin, hematocrit, fibrinogen, and C-reactive protein Age,years (CRP). Patients were followed up with $\mathrm{BMI}, \mathrm{kg} / \mathrm{m} 2$ telephone interviews for 90-days by $\mathrm{F}$ the study investigators. Patients with at FEV1 (\%) least two COPD exacerbations or one FEV1 (ml) hospitalization for AECOPD in the Smoking,n(\%) previous year were considered frequent exacerbators.

\section{Statistical analysis}

The primary outcomes were 30-day and 90-day mortality. The secondary outcome is COPD exacerbation after discharge. Categorical variables are presented as $\mathrm{n}(\%)$, and normally distributed values are presented as mean \pm standard deviation. Comparisons between groups were made using the chi-squared test (for categorical variables) or analysis of variance (for continuous variables). Receiver operator curve analysis was applied to define the minimal optimal D-dimer level and D-dimer/fibrinogen ratio that predicted death. Multivariate logistic regression analysis was applied to determine the independent factors of 30-day and

\begin{tabular}{|l|l|l|l|l|l|}
\hline Nonsmoker & $30(14.9)$ & $5(18.5)$ & ref & & \\
\hline Active smoker & $56(27.9)$ & $5(18.5)$ & 0.56 & $0.16-1.95$ & 0.564 \\
\hline Exsmoker & $115(57.2)$ & $17(63.0)$ & 0.9 & $0.33-2.45$ & 0.904 \\
\hline Smoking pack-years & $50(0-180)$ & $50(0-240)$ & 1 & $1.00-1.01$ & 0.397 \\
\hline
\end{tabular}

\section{Prognostic risk factors associated with 30-day mortality}

The mortality rate of 30-day was $5.2 \%(\mathrm{~N}: 12)$. Congestive heart failure (CHF), hypertension (HT), chronic renal failure $(\mathrm{CRF})$, peripheral vascular disease and obstructive sleep apnea syndrome (OSAS), and presence of pneumonia were related to mortality according to the univariate analysis. CCS was higher in patients who died. It was found 1-unit increment of CCS increases mortality 1.75 times. (HR: 1.75; 
Table 3. The relationship the comorbidities and 90-day (HR:1.43; $\mathrm{p}<0.04$ ), decreased sodium levels (HR:0.87; mortality

$\mathrm{p}<0.001)$ were related to increased mortality. Fibrinogen

\begin{tabular}{|l|l|l|l|l|l|}
\hline Comorbidities, $\mathrm{n}(\%)$ & $\begin{array}{l}\text { Survivors } \\
(\mathrm{N}: 201)\end{array}$ & $\begin{array}{l}\text { Non-survivors } \\
(\mathrm{N}: 27)\end{array}$ & $\mathrm{HR}$ & $\% 95 \mathrm{Cl}$ & $\mathrm{p}$-value \\
\hline CCS & $2(1-6)$ & $3(1-7)$ & 1.31 & $1.01-1.68$ & 0.451 \\
\hline Pneumoniae & $104(51.7)$ & $18(66.7)$ & 1.83 & $0.82-4.08$ & 0.137 \\
\hline Ischemic heart disease & $45(22.4)$ & $7(25.9)$ & 1.21 & $0.51-2.86$ & 0.662 \\
\hline Congestive Heart Failure & $39(19.4)$ & $8(29.6)$ & 0.05 & $0.77-4.00$ & 0.184 \\
\hline Atrial fibrilation & $16(8.0)$ & $2(7.4)$ & 1.75 & $0.22-3.93$ & 0.922 \\
\hline Hypertension & $82(40.8)$ & $17(63.0)$ & 0.93 & $1.10-5.23$ & $0.028^{*}$ \\
\hline Chronic renal failure & $10(5.0)$ & $3(11.1)$ & 2.35 & $0.71-7.81$ & 0.163 \\
\hline $\begin{array}{l}\text { Peripheral vascular } \\
\text { disease }\end{array}$ & $1(0.5)$ & $1(3.7)$ & 7.01 & $0.95-51.83$ & 0.076 \\
\hline Diabetes Mellitus & $42(20.9)$ & $9(33.3)$ & 1.84 & $0.83-4.09$ & 0.136 \\
\hline OSAS & $2(1.0)$ & $1(3.7)$ & 3.76 & $0.51-27.75$ & 0.194 \\
\hline Interstitial lung diseases & - & $1(3.7)$ & - & - & - \\
\hline
\end{tabular}

and D-dimer levels, and D-dimer/ Fibrinogen ratios were not related to 30-day mortality. Readmission to the hospital after initial exacerbation was associated with an increased risk of 30-day mortality (HR:2.70; $\mathrm{p}<0.001)$. Increased CCS (HR:1.47; $\mathrm{p}<0.05)$, readmission after an initial exacerbation (HR:1.47; $\mathrm{p}<0.05)$ were predictors for 30-day mortality in the multivariable regression model.

\section{Prognostic factors associated with 90-day mortality}

Two hundred and twenty-eight patients were followed up for 90-days and the mortality rate of 90 -day was $11.8 \%$ $(\mathrm{N}: 27)$. The relationship between demographic data and 90-day mortality has been shown in Table 2. Increased age, HT, mMRC score 4, increased CAT score were related to 90-day mortality. CCS in patients who died was significantly higher. (Table 3) Decreased RBC (HR: 0.56; p=0.014), hemoglobin (HR:0.80; $\mathrm{p}=0.007$ ), sodium (HR:0.91; $\mathrm{p}<0.01$ ) and albumin levels (HR:0.90; $\mathrm{p}=0.013)$, increased PLR (HR:1.02; $\mathrm{p}<0.03$ ) and potassium levels (HR:2.04; $\mathrm{p}=0.040)$ associated with increased 90day mortality.

D-dimer was not associated with mortality. The cut-off level of D-dimer was $1.16 \mathrm{mg} / \mathrm{L}$, but the levels above 1.16 $\mathrm{mg} / \mathrm{L}$ were not a risk factor for mortality $(p>0.05)$. In the ROC curve analysis, the cut-off level of the D-dimer/fibrinogen ratio(DDFR) was 0.11 . There was an increased risk for mortality in patients with D-dimer/fibrinogen ratios $>0.11$ and $\leq 0.11$ (HR:2.47; p <0.05). (Figure 1) D-Dimer/fibrinogen ratio was mildly correlated with CCS $(r=0,200 ; \mathrm{p}<0.01)$ without any correlation with the other clinical or laboratory parameters. It was found that a 1-unit increment of pCO2 levels increased 1.03 times the mortality of the patient who mostly has hypercarbia arterial blood gas findings (HR:1.03; p<0.05). 1-unit decrement of oxygen saturation increased the mortality (1/0.97) 1.03 times (HR:0.97; p<0.03). Decreased albumin levels (HR: 0.87; $\mathrm{p}=0.0144)$, number of exacerbations after discharge (HR:2.25; $\mathrm{p}<0.001)$ were predictive risk factors for 90 -day mortality in the multivariable regression model in which possible risk factors are included.

$\mathrm{p}<0.001)$. None of the other clinical features have been related to mortality.

Decreased hemoglobin levels (HR:0.76; $<<0.04$ ), increased Platelet/Lymphocyte ratio (PLR) (HR:1.05; p=0.005), increased creatinine (HR:8.54; $\mathrm{p}<0.01)$ and uric acid levels
Increased number of exacerbations after discharge was a predictive risk factor for 90-day mortality in the multivariable regression model (HR:2.25; p<0.001). (Table 4) Recurrent exacerbation after discharge increased the mortality risk 4.73 

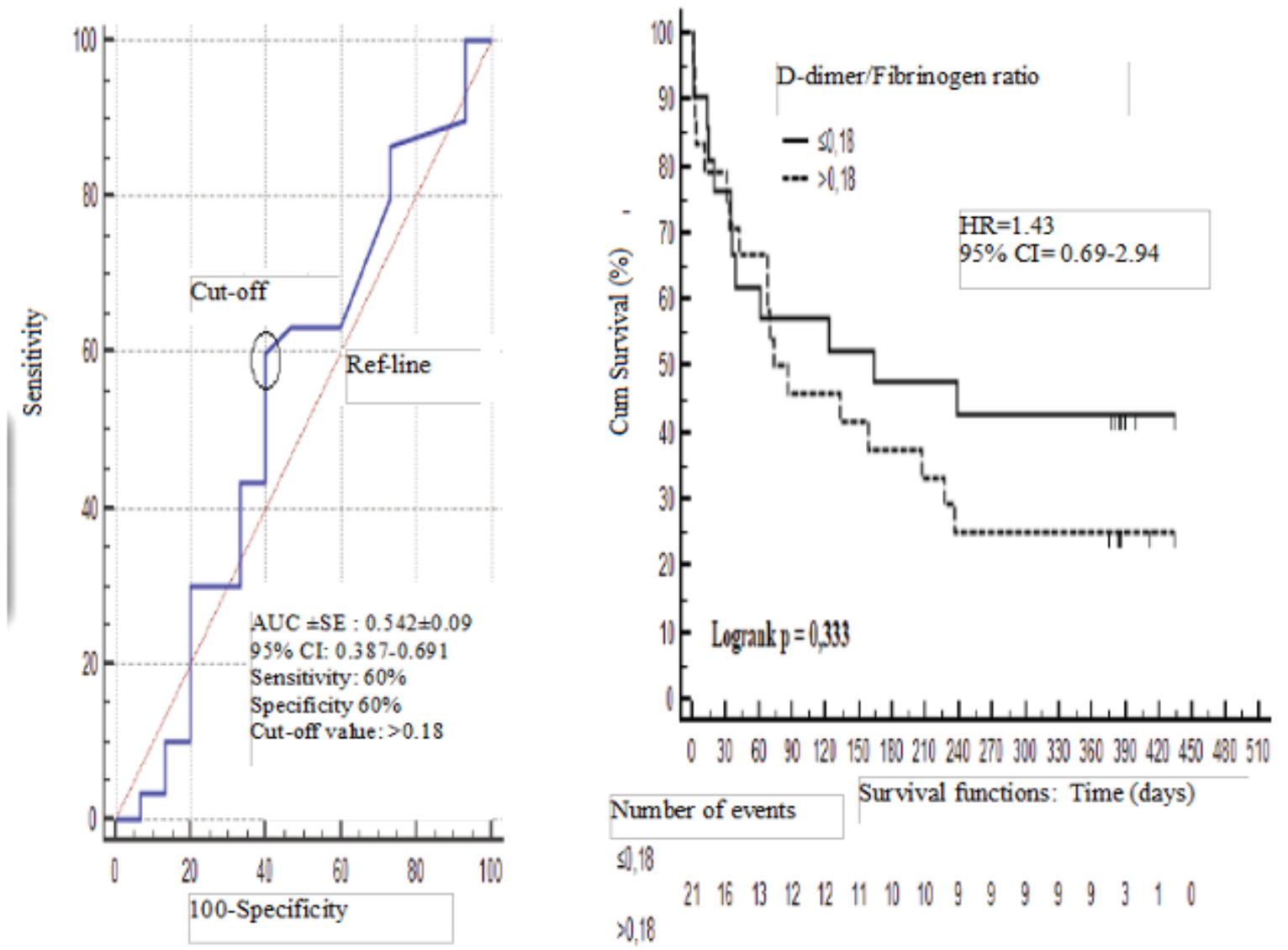

@) 18

$>0,18$

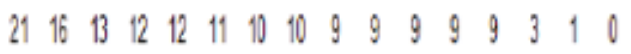

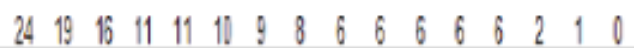

Figure 1: The predictive value of D-dimer / Fibrinogen ratio in predicting 1-year mortality and mortality risk

times (HR:4.73; p<0.01). 1-unit increment of exacerbation increased the mortality risk 3.39 times (HR:3.39; $\mathrm{p}<0.001)$.

\section{Discussion}

We showed that recurrent exacerbations after initial exacerbation were a predictor for poor prognosis of 30 day and 90-day mortality. It can be specifically concluded that recurrent exacerbation after discharge increased the mortality risk 4.73 times (HR: 4.73; p <0.01) in the 90-days periods of time. 1-unit increment of exacerbation increased the mortality risk 3.39 times (HR:3.39; $<<0.001)$. We can conclude that the $\mathrm{D}$-dimer/Fibrinogen ratio cut-off value of $>0.11$ would have a role to distinct patients as a poor and good prognosis. (HR:2.47; $\mathrm{p}<0.05)$. But D-dimer has no prognostic value in AECOPD.

In a prospectively designed study conducted by $\mathrm{Hu}$ and et $\mathrm{al}^{10}$, D-dimer $\geq 985 \mathrm{ng} / \mathrm{L}$ has been shown as a predictor for both in-hospital and 1-year mortality. But they were not regarded as 90-day mortality. In our study, we showed that D-dimer by itself does not have a prognostic role in either 30-day or 90-day mortality. In 30-day short-term mortality can be influenced by several factors including the study design as well as patient selection criteria. Controversial results for about D-dimer as a prognostic role in AECOPD can be related with the cohorts' features that include heterogeneous patients with several confounding factors. Indeed, Hu et al did not exclude diseases that could affect mortality or conditions that could lead to an increase in D-dimer ${ }^{10}$. As a result of studies conducted without excluding major associated risks that may lead to mortality, it would not be realistic to think that the mortality associated with the increase in D-dimer alone reflects COPD-related mortality. In a study designed retrospectively with the excluded confounders such as pulmonary embolism, D-dimer $>1.52 \mathrm{mg} / \mathrm{L}$ cut-off value has been conducted with 1 -year mortality in a relatively small population including 61 AECOPD patients ${ }^{9}$. But 30-day or 90-day mortality results were not reported. To determine prognostic factors associated with COPD, we excluded other important risk factors and conditions that lead to increasing D-dimer. In our study, we concluded that not D-dimer but D-dimer/Fibrinogen ratio with the cut-off value of $>0.11$ will be distinctive for the poor prognosis. D-dimer/ Fibrinogen ratio might be a potential predictor in AECOPD while none of the studies at least in our knowledge to show the exact role of this marker in COPD. Our results may encourage researchers to specifically evaluate its role in COPD as a potential prognostic marker.

We clearly showed that recurrent exacerbations after initial hospitalization would be the only independent predictor both for 30-day short-term and 90-day mortality. There are several studies which were shown that the severity and also frequency of exacerbation would be related to an increased risk of mortality ${ }^{11-14}$. Exacerbations have an increased risk of recurrence after an initial exacerbation and further deteriorate patients' health status ${ }^{15}$. We can conclude that patients hospitalized with AECOPD should be followed up closely for recurrent exacerbations who have increased risk for mortality. In such patients with recurrent exacerbations in followed 90 days should refer as "high-risk AECOPD patients" for mortality.

Recently, exacerbation history in the previous 1-year has been approved to manage treatment in $\mathrm{COPD}^{2}$. However, the results about the association between mortality risk and previous history of AECOPD were controversial ${ }^{10,13}$. Due https://dx.doi.org/10.4314/mmj.v33i4.8 
to this group of patients does not include in most of the clinical trials, it is difficult to evaluate the exact contribution to the COPD burden. Moreover, its' devastating effect on COPD outcomes is probably underestimated due to the lack of comprehensive analysis. It might be important to confirm if frequent exacerbators exacerbator was not a risk factor for mortality in $\mathrm{COPD}^{10}$. We confirmed that frequent exacerbators do not have a higher risk of mortality in both 30-day and 90-day mortality. However, we clearly showed that recurrent exacerbations after initial exacerbation were a predictor of increased mortality.

\section{Limitations of the study}

COPD is a heterogeneous disease including several comorbidities in natural history. Although several confounders accompany COPD patients in the real-life, we excluded severe diseases that could be a majör effect on mortality. We specifically excluded patients diagnosed with acute pulmonary embolism because it is a risk factor for death and may lead to an extreme increase in D-dimer levels due to aimed to show the value of $\mathrm{D}$-dimer. Thus our cohort included patients without severe diseases and acute pulmonary embolism at the time of admission to the hospital.

\section{References}

1. WHO Global Health Estimates. Available from: https://www.who. int/news-room/fact-sheets/detail/the-top-10-causes-of-death

2. Global Initiative for Chronic Obstructive Lung Disease Global Strategy for the Diagnosis, Management, and Prevention of Chronic Obstructive Pulmonary Disease. 2021.Available from: http://www. goldcopd.org/ [Google Scholar]

3. Dobric A, De Luca SN, Spencer SJ, Bozinovski S, Saling M, McDonald $\mathrm{CF}$, Vlahos R. Novel pharmacological strategies to treat cognitive dysfunction in chronic obstructive pulmonary disease. Pharmacol Ther. 2021 Oct 6:108017. doi: 10.1016/j.pharmthera.2021.108017. Epub ahead of print. PMID: 34626675.

4. Nguyen PL, Uddin MM, Mir T, Khalil A, Regmi N, Pervaiz A, Hussain T, Babu MA, Ullah I, Patel P, Lohia P, Saydain G, Koul PA, Soubani AO. Trends in Incidence, and Mortality of Acute Exacerbation of Chronic Obstructive Pulmonary Disease in the United States Emergency Department (2010-2018). COPD. 2021 Sep 16:1-9. doi: 10.1080/15412555.2021.1979500. Epub ahead of print. PMID: 34530662 .

5. Ramakrishnan S, Janssens W, Burgel PR, Contoli M, Franssen FME, Greening NJ, Greulich T, Gyselinck I, Halner A, Huerta A, Morgan RL, Quint JK, Vanfleteren LEGW, Vermeersch K, Watz H, Bafadhel M. Standardisation of Clinical Assessment, Management and Follow-Up of Acute Hospitalised Exacerbation of COPD: A Europe-Wide Consensus. Int J Chron Obstruct Pulmon Dis. 2021 Feb 16;16:321-332. doi: 10.2147/COPD.S287705. PMID: 33623379; PMCID: PMC7896731.
6. Crinion S, Cotter O, Kennedy B, O'Connor B, Curran DR, McCormack S, et al. COPD exacerbations -- a comparison of Irish data with European data from the ERS COPD Audit. Ir Med J. 2013 Oct;106(9):268, 270-2. PMID: 24416848.

7. Jacobsen PA, Kragholm KH, Torp-Pedersen C, Janssen DJA, Spruit MA, Weinreich UM. Employment Status, Readmission and Mortality After Acute Exacerbation of COPD. Int J Chron Obstruct Pulmon Dis. 2021 Aug 5;16:2257-2265. doi: 10.2147/COPD.S319840. PMID: 34385815; PMCID: PMC8352575.

8.Rizkallah J, Man SFP, Sin DD. Prevalence of pulmonary embolism in acute exacerbations of COPD: a systematic review and metaanalysis. Chest. 2009 Mar;135(3):786-793. doi: 10.1378/chest.08-1516. Epub 2008 Sep 23. PMID: 18812453.

9.Fruchter O, Yigla M, Kramer MR. D-dimer as a prognostic biomarker for mortality in chronic obstructive pulmonary disease exacerbation. Am J Med Sci. 2015 Jan;349(1):29-35. doi: 10.1097/ MAJ.0000000000000332. PMID: 25233043.

10. Hu G, Wu Y, Zhou Y, Wu Z, Wei L, Li Y, et al. Prognostic role of D-dimer for in-hospital and 1-year mortality in exacerbations of COPD. Int J Chron Obstruct Pulmon Dis. 2016 Oct 31;11:2729-2736. doi: 10.2147/COPD.S112882. PMID: 27843309; PMCID: PMC5098517.

11. MacLeod M, Papi A, Contoli M, Beghé B, Celli BR, Wedzicha JA, Fabbri LM. Chronic obstructive pulmonary disease exacerbation fundamentals: Diagnosis, treatment, prevention and disease impact. Respirology. 2021 Jun;26(6):532-551. doi: 10.1111/resp.14041. Epub 2021 Apr 24. PMID: 33893708.

12. Cardoso J, Coelho R, Rocha C, Coelho C, Semedo L, Bugalho Almeida A. Prediction of severe exacerbations and mortality in COPD: the role of exacerbation history and inspiratory capacity/total lung capacity ratio. Int J Chron Obstruct Pulmon Dis. 2018 Apr 5;13:11051113. doi: 10.2147/COPD.S155848. PMID: 29670346; PMCID: PMC5896658.

13. Celi A, Latorre M, Paggiaro P, Pistelli R. Chronic obstructive pulmonary disease: moving from symptom relief to mortality reduction. Ther Adv Chronic Dis. 2021 May 13;14:20406223211014028. doi: 10.1177/20406223211014028. PMID: 34035887; PMCID: PMC8127735.

14. García-Sanz MT, Cánive-Gómez JC, Senín-Rial L, Aboal-Viñas J, Barreiro-García A, López-Val E, et al. One-year and long-term mortality in patients hospitalized for chronic obstructive pulmonary disease. J Thorac Dis. 2017 Mar;9(3):636-645. doi: 10.21037/jtd.2017.03.34. PMID: 28449471; PMCID: PMC5394058.

15. Luo J, Zhang D, Tang W, Dou LY, Sun Y. Impact of Frailty on the Risk of Exacerbations and All-Cause Mortality in Elderly Patients with Stable Chronic Obstructive Pulmonary Disease. Clin Interv Aging. 2021 Apr 13;16:593-601. doi: 10.2147/CIA.S303852. PMID: 33880018; PMCID: PMC8053481. 